\title{
THE INFLUENCE OF EXPOSURE PARAMETERS ON THE QUALITY OF THE PARTS BUILT BY SELECTIVE LASER PROTOTYPING
}

\author{
Stanca Comsa ${ }^{1}$, Theodor Cezar Milian², Doina Gheorghiu ${ }^{3}$ \\ 1,2,3 INCDMTM-Bucharest \\ stanca comsa@yahoo.com, miliantheodor@yahoo.com, doina gh@yahoo.com
}

\begin{abstract}
This paper examines the influence of exposure parameters: scanning speed, laser power, laser beam offset, scan type trajectory on the quality of the parts built by the selective laser sintering of metal powders, used in EOSINT M270 M Dual Mode prototyping equipment. For this analysis, a series of parts with different geometries were made, and the dimensional precision along with surface quality were analysed. In conclusion, depending on the purpose (better surface quality or increased hardness), it is possible to choose which work parameter needs to be modified in order to obtain the desired result.
\end{abstract}

Keywords: rapid prototyping \& manufacturing, selective laser sintering, 3D metal printing

\section{Introduction}

Selective laser prototyping: Direct Metal Laser Sintering (DMLS).

This technology uses a high-power laser (200 watts for the equipment used) to melt the metallic powder layer by layer. Using this method, it is possible to print custom medical guides, consolidated airspace parts, deep slots that would not be achieved by other processes (casting/machining) thus saving time, energy and costs.

A wide range of metal alloy powders: Titanium 64, CoCr, Inconel, Aluminium, for sintering on the EOSINT 270 M Dual Mode equipment [1], belonging to the BIOMECHATRONIC Laboratory, part of the National Institute of Research and Development in Mechatronics and Measurement Technique, Bucharest. (Figure 1).

By acquiring this equipment, INCDMTM has implemented an advanced technology for making prototypes and complex metal powder samples by selective laser sintering.

\section{Data Preparation Software}

The 3D virtual models of the parts designed in SolidWorks were saved in a Standard Tessellation Language (. STL) file and then imported into Magics RP software from Materialise company [2]. This software is used to multiply, orient and position the parts on the print surface, add the supports, and prepare the job to be exported to the machine.

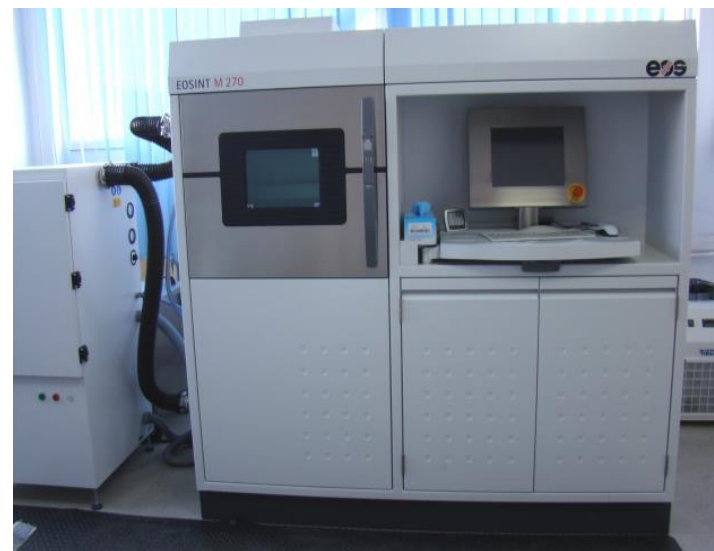

Figure 1: EOSINT M270 Dual Mode, owned by INCDMTM

The supports are required to be strong enough to avoid detachment of parts from the baseplate during the job due to uneven contraction, and transfer the heat to it.

In the next step, the parts with the supports saved in the STL file are pre-processed using the EOS RP Tools program, and converted into a SLI 2D file type. (Figure 2). This conversion is necessary because sintering occurs only through melting layer by layer.

The virtual 3D model and the supports are divided into layers with a thickness of 0.02 or $0.03 \mathrm{~mm}$ depending on the material used.

After "slicing" the part, an error checking must be carried out to correct any errors in the file. The operation is necessary as parts made in $3 \mathrm{D}$ design software may have some errors, often undetectable by the user, that can lead to problems in the printing operation. 


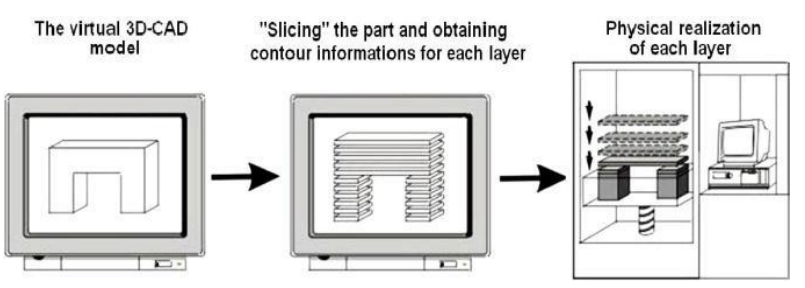

Figure 2: Conversion of 3D STL file in 2D SLI file

The next step is to import the parts in the M270 machine system process software (PSW). A very useful option of the PSW software is the EOS Parameter Editor which allows the exposure parameters to be changed for each part/support in the same job.

\section{Building parts with different parameters}

In order to determine how the process parameters, affect the dimensional and tolerances of the assembly, the structural characteristics, the surface quality and the possibility of detaching from the construction plate, a series of parts was made in 3 jobs.

The first job involves building parts with different scanning speeds and laser power settings, as shown in Figure 3.

The four-part sets made within this job had the following parameters:

- Batch 1: Default CoCr Powder Machine Settings;

- Batch 2: Parts built with lower laser beam power (the default is $195 \mathrm{~W}$, but $150 \mathrm{~W}$ was used);

- Batch 3: Parts built with lower laser beam scanning speed (default $1500 \mathrm{~mm} / \mathrm{s}$, $1000 \mathrm{~mm} / \mathrm{s}$ used).

- Batch 4: Parts built with higher beam scanning speed (default $1500 \mathrm{~mm} / \mathrm{s}$, $2000 \mathrm{~mm} / \mathrm{s}$ used).

Job 2 shown in Figure 4, consisted of 6 types of parts with different geometries.

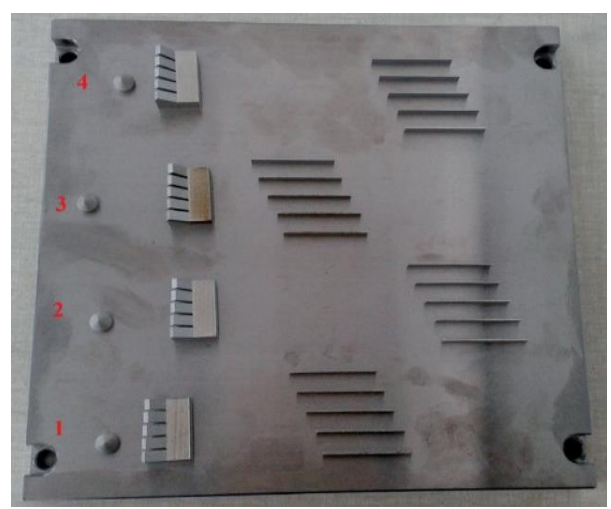

Figure 3: Parts printed on the baseplate in job 1.
In this job was modified the scanning distance and direction as well as the number of post contours made on the parts for noted their influence on the surface quality and hardness:

- Batch 1: Default settings for CoCr powder;

- Batch 2: Parts with increased scanning distance $(0.2 \mathrm{~mm})$;

- Batch 3: Parts built with less scanning distance $(0.05 \mathrm{~mm})$;

- Batch 4: Parts made with 2 post contours and 150W laser beam power;

- Batch 5: Parts made while keeping laser beam scanning direction only along the $\mathrm{X}$ axis;

- Batch 6: A single small part with modified supports to ensure firm fixation on the baseplate.

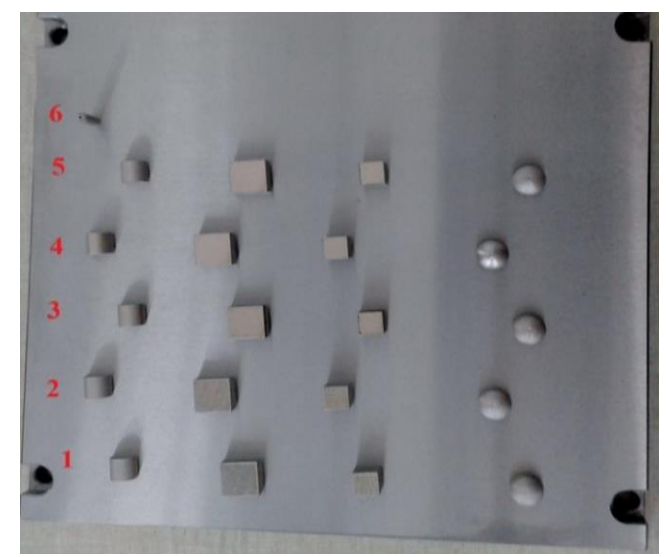

Figure 4: Arrangement of the parts on the baseplate in job 2

The type of parts built in job3 are shown in Figure 5.

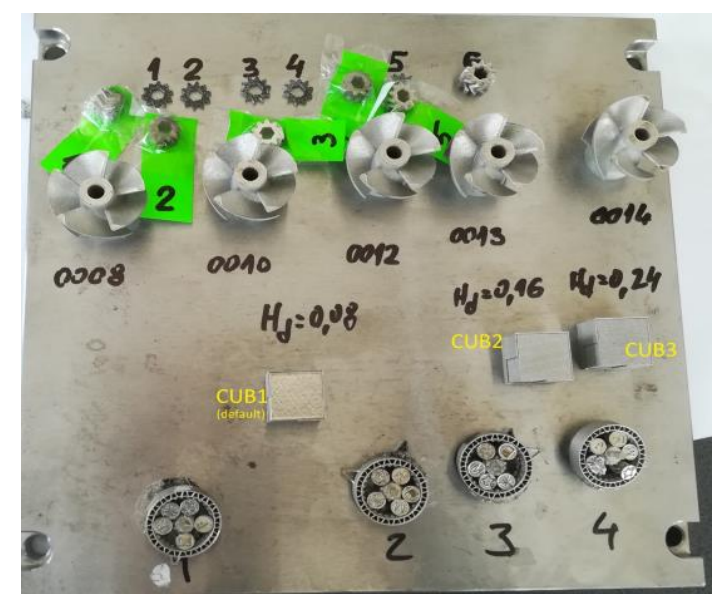

Figure 5: The baseplate with the printed parts in job3 and their encoding

The first row of parts (those at the top of the construction board) are the gears.

In this set of parts, the parameters were modified for making the supports on the construction plate according to Table 1 . 
Table 1: Exposure parameters for gearwheel

\begin{tabular}{|c|c|c|c|c|c|c|c|}
\hline \multicolumn{2}{|c|}{ Part number } & $\mathbf{1}$ & $\mathbf{2}$ & $\mathbf{3}$ & $\mathbf{4}$ & $\mathbf{5}$ & $\mathbf{6}$ \\
\hline $\begin{array}{c}\text { Coordinate on } \\
\text { baseplate }\end{array}$ & $\mathrm{x}[\mathrm{mm}]$ & 26 & 40 & 64 & 81 & 121 & 151 \\
\cline { 2 - 8 } & $\mathrm{y}[\mathrm{mm}]$ & 193 & 193 & 196 & 196 & 199 & 201 \\
\hline \multicolumn{2}{|c|}{ Laser Power [W] } & 90 & 90 & 90 & 90 & 90 & 120 \\
\hline \multicolumn{2}{|c|}{ Scan speed [mm/s] } & 1350 & 900 & 450 & 300 & 200 & 200 \\
\hline $\begin{array}{c}\text { Part exposure parameters } \\
\text { type }\end{array}$ & \multicolumn{6}{c|}{ EOS_DirectPart } \\
\hline
\end{tabular}

In the second series of parts (fan rotor type) it was observed the relation between the exposure parameters and the surface quality.
The modified parameters associated with each part of this series are specified in Table 2 .

Table 2: Exposure parameters for fan rotor

\begin{tabular}{|c|c|c|c|c|c|}
\hline Part number & 0008 & 0010 & 0012 & 0013 & 0014 \\
\hline \multirow{2}{*}{$\begin{array}{l}\text { Coordinate on } \\
\text { baseplate }\end{array}$} & 2 & 56 & 108 & 146 & 200 \\
\hline & 130 & 138 & 148 & 149 & 157 \\
\hline \multicolumn{6}{|l|}{ Set of parameters for the shape } \\
\hline Laser Power [W] & 100 & 100 & 70 & 160 & 100 \\
\hline Scan speed $[\mathrm{mm} / \mathrm{s}]$ & 300 & 1200 & 600 & 600 & 600 \\
\hline \multicolumn{6}{|l|}{ Parameter set for Downskin } \\
\hline Laser Power [W] & 100 & 100 & 70 & 160 & 100 \\
\hline Scan speed $[\mathrm{mm} / \mathrm{s}]$ & 1200 & 4800 & 2400 & 2400 & 2400 \\
\hline Exposure type for (skin) & \multicolumn{5}{|c|}{ _Default_OuterSkin_Directpart } \\
\hline Exposure type for part (Core) & \multicolumn{5}{|c|}{ Default_innerSkin } \\
\hline Exposure type for Postcontour & \multicolumn{5}{|c|}{ _Default_Postcontours } \\
\hline Contour thickness (z) [mm] & \multicolumn{5}{|c|}{3} \\
\hline $\begin{array}{l}\text { Support radius for baseplate } \\
{[\mathrm{mm}]}\end{array}$ & \multicolumn{5}{|c|}{0} \\
\hline
\end{tabular}

The parts were placed in different areas of the building volume so as to track the influence of their positioning on the precision of execution. The $\mathrm{x}, \mathrm{y}$ coordinates of the 6 parts represent the position of the centre of the parts on the baseplate.
For the third series of parts (cubes) it was observed how the changing of parameters affect surface quality. Parameters associated with each part of this set are shown in Table 3.

Table 3: Exposure parameters for cubes

\begin{tabular}{|c|c|c|c|}
\hline Part number & Cube1 default) & Cube2 & Cube3 \\
\hline \multirow{2}{*}{\begin{tabular}{ll|l|}
$\begin{array}{l}\text { Coordinates } \\
\text { baseplate }\end{array}$ & on & $\mathrm{x}[\mathrm{mm}]$ \\
\cline { 2 - 2 } & $\mathrm{y}[\mathrm{mm}]$ \\
\end{tabular}} & 80 & 170 & 200 \\
\hline & 57 & 73 & 80 \\
\hline \multirow[t]{2}{*}{ Exposure Parameters } & \multicolumn{3}{|c|}{ Exposure name type } \\
\hline & $\begin{array}{c}\text { Default_outerSkin } \\
\text { _Directpart }\end{array}$ & OSHD_016 & OSHD_024 \\
\hline Hatch Distance[mm] & 0,08 & 0,16 & 0,24 \\
\hline Scan speed[mm/s] & \multicolumn{3}{|c|}{800} \\
\hline Laser Power[W] & \multicolumn{3}{|c|}{195} \\
\hline Beam Offset[mm] & \multicolumn{3}{|c|}{0,03} \\
\hline Stripes width[mm] & \multicolumn{3}{|c|}{4} \\
\hline Stripes overlap[mm] & \multicolumn{3}{|c|}{0,10} \\
\hline Skywriting & \multicolumn{3}{|c|}{ On } \\
\hline Stripes alternating $\mathrm{x}, \mathrm{y}$, & \multicolumn{3}{|c|}{ On } \\
\hline $\begin{array}{l}\text { Exposure } \\
\text { parameters for: }\end{array}$ & Upskin & \multicolumn{2}{|c|}{ Downskin } \\
\hline Distance $[\mathrm{mm}]$ & \multicolumn{3}{|c|}{0,05} \\
\hline Scan speed[mm/s] & 800 & \multicolumn{2}{|c|}{3000} \\
\hline Laser Power[W] & \multicolumn{3}{|c|}{195} \\
\hline Thickness $[\mathrm{mm}]$ & \multicolumn{3}{|c|}{0} \\
\hline Overlap with inskin[mm] & \multicolumn{3}{|c|}{0,10} \\
\hline Minimum length [mm] & \multicolumn{3}{|c|}{2} \\
\hline
\end{tabular}


In the fourth series of parts (planetary gears), the laser beam offset parameter for each part was modified, and the assembly tolerance monitored.
Parameters associated with each part of this set are shown in Table 4.

Table 4: Exposure parameters for Planetary gears

\begin{tabular}{|l|l|c|c|c|c|c|}
\hline Part number & $\mathbf{1}$ & $\mathbf{2}$ & $\mathbf{3}$ & $\mathbf{4}$ & $\mathbf{5}$ \\
\hline $\begin{array}{l}\text { Coordinates on } \\
\text { baseplate }\end{array}$ & $\mathrm{x}[\mathrm{mm}]$ & 62 & 102 & 141 & 183 \\
\cline { 2 - 7 } & $\mathrm{y}[\mathrm{mm}]$ & 10 & 14 & 19 & 24 & 29 \\
\hline General Beam Offset - GBO [mm] & \multicolumn{5}{|c|}{0,09} \\
\hline Part Beam Offset - PBO[mm] & 0 & $-0,02$ & $\begin{array}{c}+0,0 \\
2\end{array}$ & $+0,05$ & $+0,1$ \\
\hline Supports & \multicolumn{5}{|c|}{450} \\
\hline Scan speed [mm/s] & \multicolumn{5}{|c|}{90} \\
\hline Laser power [W] & \multicolumn{5}{|c|}{} \\
\hline
\end{tabular}

\section{Results}

Following the visual inspection of the parts performed in the first job, it was observed that the parts built with low scan speed were overexposed (brown-coloured surfaces), and those made with higher scanning speeds of the laser beam had a lower roughness.

Overexposure was also observed in the parts built in job 2 to reduce the scanning distance. By strengthening the supports, the small part of job 2 did not detach from the baseplate during processing. Measurements were made on the parts built in job 2, for a quantitative analysis of the influence of parameters on the roughness and hardness.

The roughness measurements were performed with IND 120 - TAYLOR HOBSON Rugosimeter from the INCDMTM Length Laboratory [3], which has the ability to measure roughness, curl and contour simultaneously, both on flat surfaces and on curved surfaces, being designed for small parts. The measurements are made according to ISO 4287/2003. [4]

The hardness measurement was performed with the hardness tester 251 VRSA-AFFRI/Model 206X from the INCDMTM Lengths Laboratory for Small and Medium Metal Parts. The measurements are made according to SR EN ISO 6507-1 / 2006. [5]

The results of the measurements, both for roughness and for hardness, are presented in Table 5 .

'Table 5: Measurement results for parts from job 2

\begin{tabular}{|c|c|c|c|c|c|}
\hline Lot & Part & $\begin{array}{l}\text { Rmin } \\
(\mu \mathrm{m})\end{array}$ & $\begin{array}{c}\text { Rmax } \\
(\mu \mathrm{m})\end{array}$ & $\begin{array}{c}\mathrm{Ra} \\
(\mu \mathrm{m})\end{array}$ & $\begin{array}{c}\text { Hardness } \\
\text { HRC }\end{array}$ \\
\hline \multirow[t]{4}{*}{1} & Cube 1 & $-4,5$ & 8,5 & 1,1288 & 39,0 \\
\hline & Parallelepiped 1 & -16 & 12 & 3,1808 & 42,0 \\
\hline & Spherical calotte 1 & -23 & 41 & 7,9985 & \\
\hline & Half-cylinder 1 & $-10,5$ & 9 & 4,4268 & 44,5 \\
\hline \multirow[t]{4}{*}{2} & Cube 2 & $-3,3$ & 3,2 & 0,9389 & 31,0 \\
\hline & Parallelepiped 2 & -12 & 9 & 3,1428 & 41,6 \\
\hline & Spherical calotte 2 & -31 & 41 & 8,5330 & \\
\hline & Half-cylinder 2 & -8 & 8 & 3,9338 & 35,0 \\
\hline \multirow[t]{4}{*}{3} & Cube 3 & -11 & 5 & 1,6255 & 33,0 \\
\hline & Parallelepiped 3 & -15 & 16 & 4,5010 & 45,0 \\
\hline & Spherical calotte 3 & -31 & 28 & 6,3227 & \\
\hline & Half-cylinder 3 & $-11,5$ & 8 & 3,9161 & 41,0 \\
\hline \multirow[t]{4}{*}{4} & Cube 4 & $-7,5$ & 13 & 1,3255 & 38,0 \\
\hline & Parallelepiped 4 & -7 & 8,5 & 2,6485 & 44,0 \\
\hline & Spherical calotte 4 & $-19,5$ & 29 & 4,3105 & \\
\hline & Half-cylinder 4 & -7 & 8,5 & 3,4594 & 38,5 \\
\hline \multirow[t]{4}{*}{5} & Cube 5 & $-4,2$ & 3,5 & 1,0648 & 39,0 \\
\hline & Parallelepiped 5 & -11 & 11 & 3,2367 & 44,0 \\
\hline & Spherical calotte 5 & -23 & 28 & 6,4980 & \\
\hline & Half-cylinder 5 & -10 & 8 & 3,4520 & 45,0 \\
\hline
\end{tabular}

For a clearer understanding of the roughness variation, depending on the machine's working parameters and the shape and size of the surface, the roughness values $\mathrm{Ra}(\mu \mathrm{m})$ presented in Table 5 were plotted for easier comparison in Figure 6 and 7.
The analysis of the diagram in Figure 7 reveals that the parts belonging to batch 3 , made with the decrease of the surface hatch width, have the highest roughness, so this way of changing the parameters should be avoided in practice, if smooth surfaces are desired. 


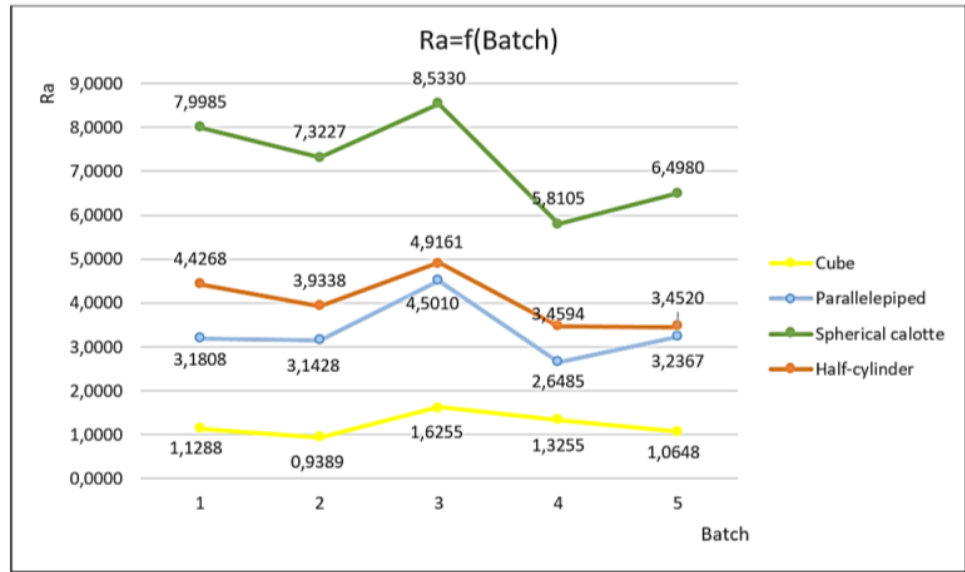

Figure 6. Roughness depending on production batch

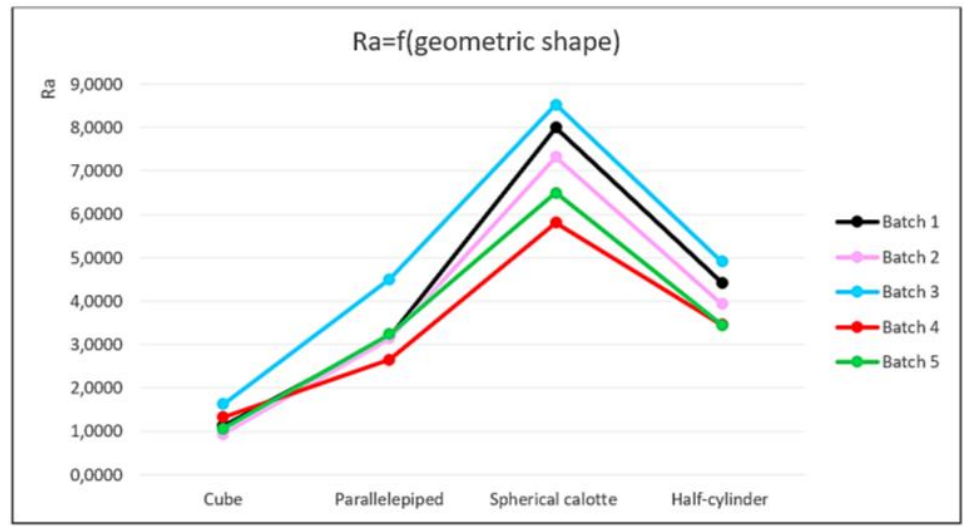

Figure 7: Roughness depending on geometric shape

The maximum roughness appeared as expected in the curved surfaces, namely the spherical cap and the half-cylinder. This phenomenon is normal and is due to prototyping technology, which involves slicing the parts in successive layers, which means that the curved surfaces show the so-called "ladder" effect, which automatically leads to the increase of the roughness of the parts.

This effect is all the more important as the surface is curved in more than one direction and so, as can be seen in Figure 6 , the roughness of the spherical cap is greater than that of the half-cylinder.
The ruggedness of planar surfaces parallel to the surface of the baseplate is the smallest. It can also be concluded that the surface size also influences the Ra roughness value.

The best part roughness was obtained for batch 2 and batch 4 respectively, which used the following working parameters:

- Batch 2: Parts made by increasing the hatching distance of the surface to $0.2 \mathrm{~mm}$;

- Batch 4: Parts made with 2 post contours and 150W laser beam power.

The effect of the parameters on the hardness of the parts, is shown in Figure 8.

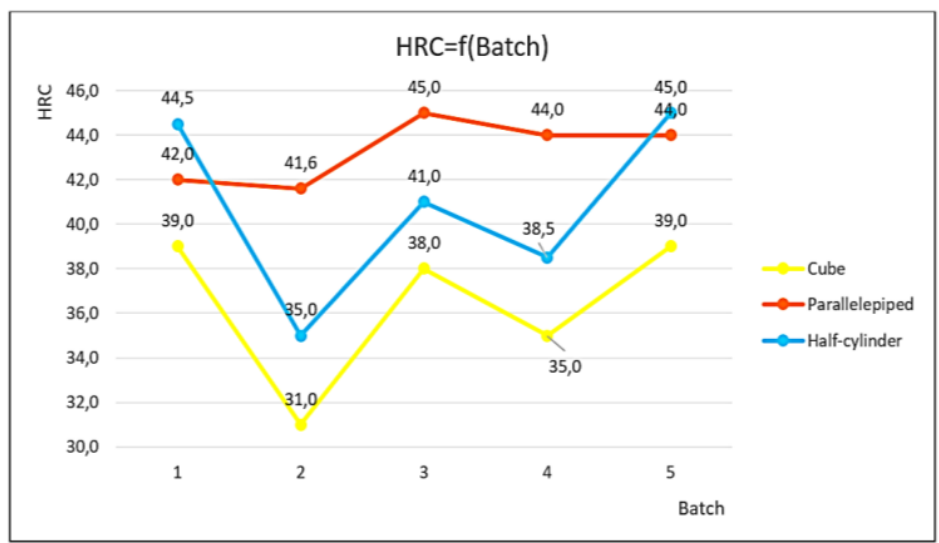

Figure 8: Hardness depending on the manufacturing batch 
It should be noted that the spherical calotte was excluded from this study because the measuring device showed errors in testing. It is remarked from the diagram that the way of variation of hardness is almost identical in the case of cube, parallelepiped and similar to the hardness measured on the half cylindrical part. Differences are not spectacular, but the highest hardness is obtained for batch 1 and 5 . Similar results are also obtained for batch 3 , in which overexposure leads to the superior quality of the executed parts.

For the first row of parts in job 3, it was measured the force needed for detaching the parts, depending on the exposure parameters for the supports. They should not allow accidental detachment of parts during the process but be easily removed by post processing. A torque wrench was used to detach the parts from the baseplate and the breaking torque for the supports was measured. The supports for parts 1, 2 and 3 was broken very easily, for the parts 4 and 5 was needed a larger torque, and part 6 could not be detached with the key and required cutting off with the saw.

The results obtained are presented in Table 6

Table 6: Breaking torque for supports

\begin{tabular}{|c|c|c|c|c|c|c|}
\hline Part number & $\mathbf{1}$ & $\mathbf{2}$ & $\mathbf{3}$ & $\mathbf{4}$ & $\mathbf{5}$ & $\mathbf{6}$ \\
\hline Breaking torque[Nm] & 1 & 3 & 5 & 10 & 30 & - \\
\hline
\end{tabular}

The quality of the upper and lower surfaces after the detachment of the supports can be seen in Figure 9.

From the analysis of the dimensions in the 2 nd row of the $3 \mathrm{rd} \mathrm{job}$, the positioning of the parts on the construction plate does not influence the accuracy of their execution, the differences being insignificant.

Increasing the scanning distance within the line 3 of job 3 leads to increased surface roughness. However, the execution time is shorter.

From the analysis of the results obtained at the change of the laser part beam offset (PBO) it can be noticed that the tolerances in the planetary gearboxes (row 4 of job 3) varies inversely with it.

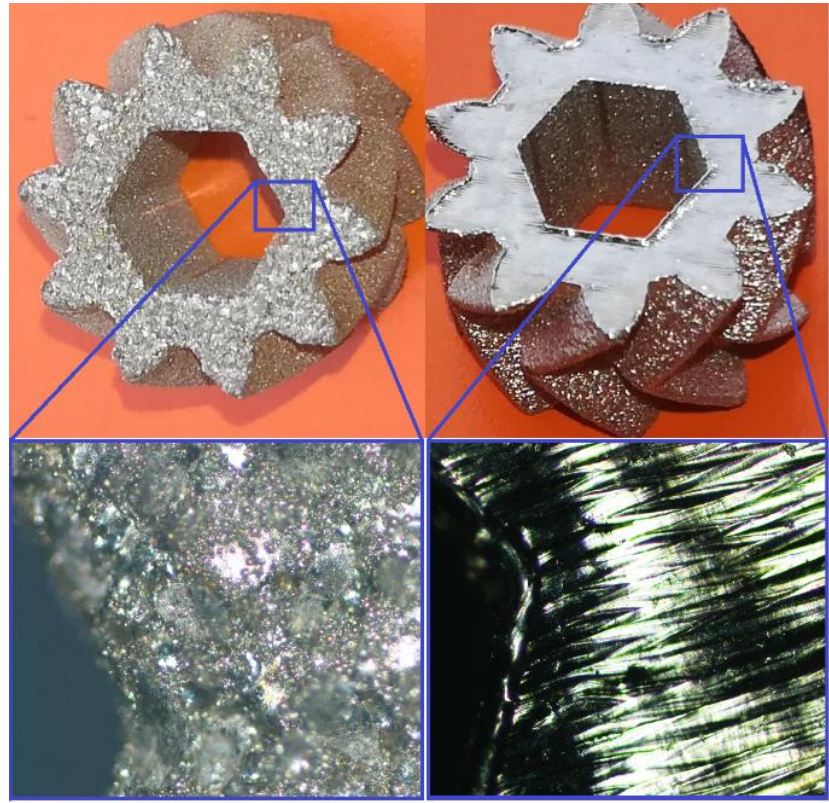

Figure 9: The gearwheel surfaces for down skin: where the supports make contact with the part (left), and for up skin (right picture)

\section{Acknowledgements}

This work has been funded by National Institute for Research and Development in Mechatronics and Measurement Technique through projects:

- "Advanced research on the use and optimization of selective laser sintering processes with application in the field of human tissues and prosthesis and extension in biomechatronic field" Nucleus Program Contract number 3N/2016- PN 16 210501 ;

- „3D printing technology evaluation for turbopump rotor manufacturing"- 3dRotor STAR Program Contract number 138/20.07.2017.

\section{References}

\section{[1] www.eos.info}

[2] www.materialise.com

[3] www.incdmtm.ro

[4] ISO 4287/2003 - Geometrical Product Specifications (GPS) - Surface texture: Profile method - Terms, definitions and surface texture parameters.

[5] ISO 6507-1:2005: Metallic materials - Vickers hardness test - Part 1: Test method. 